\title{
Online Purchase Prediction via Multi-Scale Modeling of Behavior Dynamics
}

\author{
Chao Huang* \\ University of Notre Dame, JD Digits \\ chaohuang75@gmail.com \\ Chuxu Zhang \\ University of Notre Dame \\ czhang11@nd.edu
}

\author{
Xian $\mathrm{Wu}^{*}$ \\ University of Notre Dame \\ xwu9@nd.edu \\ Jiashu Zhao, Dawei Yin \\ JD.com \\ zhaojiashu1@jd.com,yindawei@acm.
}

\author{
Xuchao Zhang \\ Virginia Tech \\ xuczhang@vt.edu \\ Nitesh V. Chawla \\ University of Notre Dame \\ nchawla@nd.edu
}

\begin{abstract}
Online purchase forecasting is of great importance in e-commerce platforms, which is the basis of how to present personalized interesting product lists to individual customers. However, predicting online purchases is not trivial as it is influenced by many factors including: (i) the complex temporal pattern with hierarchical inter-correlations; (ii) arbitrary category dependencies. To address these factors, we develop a Graph Multi-Scale Pyramid Networks (GMP) framework to fully exploit users' latent behavioral patterns with both multi-scale temporal dynamics and arbitrary inter-dependencies among product categories. In GMP, we first design a multi-scale pyramid modulation network architecture which seamlessly preserves the underlying hierarchical temporal factorsgoverning users' purchase behaviors. Then, we employ convolution recurrent neural network to encode the categorical temporal pattern at each scale. After that, we develop a resolution-wise recalibration gating mechanism to automatically re-weight the importance of each scale-view representations. Finally, a context-graph neural network module is proposed to adaptively uncover complex dependencies among category-specific purchases. Extensive experiments on real-world e-commerce datasets demonstrate the superior performance of our method over state-of-the-art baselines across various settings.
\end{abstract}

\section{CCS CONCEPTS}

- Information systems $\rightarrow$ Web mining; Recommender systems;

*These authors contributed equally. This work was done when Chao Huang was a Ph.D. student at University of Notre Dame

Permission to make digital or hard copies of all or part of this work for personal or classroom use is granted without fee provided that copies are not made or distributed for profit or commercial advantage and that copies bear this notice and the full citation on the first page. Copyrights for components of this work owned by others than the author(s) must be honored. Abstracting with credit is permitted. To copy otherwise, or republish, to post on servers or to redistribute to lists, requires prior specific permission and/or a fee. Request permissions from permissions@acm.org.

KDD '19, August 4-8, 2019, Anchorage, AK, USA

(C) 2019 Copyright held by the owner/author(s). Publication rights licensed to the Association for Computing Machinery.

ACM ISBN 978-1-4503-6201-6/19/08 ..\$15.00

https://doi.org/10.1145/3292500.3330790

\section{KEYWORDS}

Purchase Prediction; Temporal Dynamics; Recommendation Systems; Deep Neural Networks

ACM Reference Format:

Chao Huang, Xian Wu, Xuchao Zhang, Chuxu Zhang, Jiashu Zhao, Dawei Yin, and Nitesh V. Chawla. 2019. Online Purchase Prediction via MultiScale Modeling of Behavior Dynamics. In The 25th ACM SIGKDD Conference on Knowledge Discovery and Data Mining (KDD '19), August 4-8, 2019, Anchorage, AK, USA. ACM, New York, NY, USA, 10 pages. https: //doi.org/10.1145/3292500.3330790

\section{INTRODUCTION}

Online purchase prediction is of great importance for a wide spectrum of user-centric applications in online retailing platforms, ranging from personalized recommender systems [2], user activity modeling [38] and resource management [6]. For instance, by knowing the categories of products, customers tend to purchase on a daily basis, online retailers can: (i) alleviate the information overload issue and help customers to meet a variety of their needs and tastes [34]; (ii) increase the profit for online retailers through managing the traffic [28]. Hence, making predictions on customers' future online purchases is key to enhancing the experience and satisfaction of customers and online retailers. To facilitate this task, we seek to develop effective predictive models with the goal of forecasting purchase behavior of customers on each product category at the future time step given their historical purchase records.

Intuitively, we can employ conventional time series forecasting techniques. However, the purchase sequences involve dynamic and non-linear temporal dependencies across time steps, which pose difficulties to many existing time series forecasting models-relying on the stationary and linear assumption of time series data, such as autoregressive integrated moving average (ARIMA) [18] and its variants (e.g., seasonal-ARIMA [15]). To mitigate this issue, various types of non-linear deep neural network models (e.g., LSTM and GRU) have been introduced to consider the time-varying sequential patterns. Nevertheless, a common drawback of the above approaches is that only one dimensional temporal dynamics is considered, which may not properly reflect the variation in many real world scenarios. In fact, real-world temporal patterns of customer purchase behavior are much more complicated, involving daily routines, weekly pattern, monthly periodicity, and even other personalized periodic transition regularities, which naturally form a 
type of multi-scale temporal dynamics. For example, in the scenarios that product lifespan usually vary among different categories (e.g., daily necessities, electronic devices), purchase behavioral data is often exhibited with both time-dependent and multi-dimensional dependencies. This sheds light on the weakness of existing time series forecasting methods, and motivates us to capture multi-scale temporal dynamics in modeling customers' purchase behavior that can result in more accurate prediction results.

In addition to the importance of considering multi-scale temporal patterns of online purchase behavior, another key dimension is to understand dependencies among product categories, to augment predictions with relevant context signals [25]. In real life, explicit and implicit dependencies among product categories are ubiquitous when users make online purchases [23]. For example, when a user in an online store is examining sports clothes for athletic activities, he/she might also be interested in buying fitness foods which are good for weight loss and building muscle. People may buy forks/plates and beer together for holding a party. In such cases, purchases on different categories are no longer independent. If the context-aware relationships among categories are ignored and each individual category-specific purchases are treated as independent ones, it is likely that the modeling of users' purchase behavior is inaccurate, and thus the prediction performance is degraded.

We identify two key challenges of modeling online purchase behavior, which motivate the model design (as illustrated in Figure 1).

Temporal Pattern Fusion with Hierarchical Inter-Correlations. It is a significant challenge to learn a temporal representation which can comprehensively reflect time-ordered sequential patterns of users' purchase behavior from different scales (resolutions). Different scale views (e.g., daily, weekly, bi-weekly, monthly) usually provide complementary information for modeling online purchase activities [34]. Additionally, each scale view may exhibit pairwise correlations or even higher-order cross-resolution correlations, and can be represented in a hierarchical manner. If features are learned from different views separately and then loosely couple them together by directly concatenating the feature embeddings as the final representation, it cannot capture the cross-scale correlations and preserve the semantic multi-scale structural information of online purchase data. Hence, in order to learn meaningful purchase representations from long, broad and hierarchical temporal inputs, a robust across-scale temporal feature learning model with effective pattern fusion mechanism is needed in our studied problem.

Dynamic and Arbitrary Category Dependencies. Distinct to stationary inter-dependent relations, the process of inter-category influences on online purchase behavior is rather dynamic [27], since users' purchase preferences could change over time (e.g., women may be no longer interested in pregnancy products after the childbirth). Hence, prediction techniques for static scenarios can hardly be responsive to dynamic changes. Additionally, the dependencies across categories can be arbitrary since any pair of category-specific purchases could potentially be related in various online shopping scenarios [37]. For instance, a user can order snacks from an online store for a social party or outdoor activities. Users often make correlated purchases and exhibit different dependencies in choosing items of different categories due to his/her specialty. Therefore, to build effective online purchase predictive models with

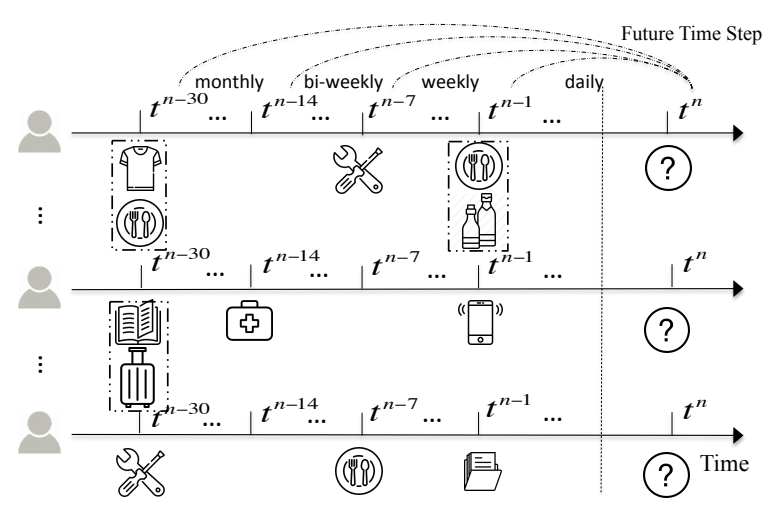

Figure 1: Illustration of the online purchase prediction problem with multi-scale temporal dynamics and arbitrary category dependencies.

the context of category dependencies, it is crucial to generalize our framework to jointly capture dynamic and arbitrary category correlation structures.

To address the aforementioned challenges, this work proposes a general framework-Graph Multi-Scale Pyramid Networks (GMP)for online purchase prediction. Specifically, at the first stage, we design a multi-scale pyramid modulation network to model multiresolution temporal factors that govern the sequential regularities of users' purchase behavior. Then, we leverage convolution recurrent networks to encode multi-dimensional temporal patterns separately from low-level (locally) to high-level (globally). At the second stage, a resolution-wise recalibration gating mechanism is developed to promote the collaboration across different resolution views, and automatically capture the importance of contributed temporal patterns. Finally, a context-graph neural network module is proposed to handle dynamic dependencies among category-specific purchases, which is able to adaptively aggregate global contextual information when modeling complementary purchase behavior.

In summary, we highlight our contributions as follows:

- We introduce a novel multi-scale pyramid modulation network architecture for predicting users' online purchases, which can explore the high-order and time-dependent structural correlations underlying multi-scale temporal dynamics of online purchase behavior in a hierarchical way.

- We develop a resolution-wise recalibration gating mechanism for fusing scale-specific pattern representations, and automatically capturing the importance of each scale-view in the predictive model. In addition, we propose a context-graph neural network module which is capable of uncovering dynamic dependencies among categories.

- Extensive experiments on three real-world e-commerce datasets are performed along with comparisons to existing state-of-theart predictive models, to demonstrate the advantages of our GMP model across various settings.

\section{PROBLEM FORMULATION}

In this section, we begin with some necessary notations and then formally present the online purchase forecasting problem in this paper. Suppose we have $M$ users $U=\left\{u_{1}, \ldots, u_{m}, \ldots, u_{M}\right\}(1 \leq m \leq$ $M)$ and $N$ project categories $C=\left\{c_{1}, \ldots, c_{n}, \ldots, c_{N}\right\}(1 \leq n \leq N)$. 
We refer to an individual user as $u_{m} \in U$, an individual product category as $c_{n} \in C$, where $m, n$ and $t$ are defined as the index for the user, category and time step, respectively.

Definition 1. Purchase Matrix $\mathcal{X}_{m}$. Given a window of T time steps (e.g., day), we define a matrix $\mathcal{X}_{m} \in \mathbb{R}^{N \times T}$ to represent the purchase records of each user $u_{m}$ on each category $c_{n} \in C$ over time. The $(n, t)$-th entry of $X^{m}$ is denoted as $x_{m, n}^{t}$. In particular, $x_{m, n}^{t}=1$ if the $m$-th user purchased items of $n$-th category at the $t$-th time step and $x_{m, n}^{t}=0$ otherwise.

Problem Statement. With the aforementioned definitions, online purchase prediction problem can be formulated as: given the purchase behavior data of each user (i.e., $\mathcal{X}_{m}$ ) from previous $T$ time steps, the objective is to learn a mapping function which predicts the unknown purchase behaviors of each user $u_{m}$ on each product category $c_{n}$ in the future time steps (i.e., $x_{m, n}^{(T+1)}$ ).

$$
x_{m}^{(T+1)}=F\left(\mathcal{X}_{m} \in \mathbb{R}^{N \times T}\right) ; \quad\left(u_{m} \in U\right)
$$

where $F(\cdot)$ is the mapping function we aim to learn, $x_{m}^{(T+1)}=$ $\left\{x_{m, 1}^{(T+1)}, \ldots, x_{m, n}^{(T+1)}, \ldots, x_{m, N}^{(T+1)}\right\}$.

\section{METHODOLOGY}

Generally speaking, our proposed GMP model consists of four key components (as shown in Figure 4): a) modeling temporal hierarchy with a multi-scale pyramid modulation network; b) learning resolution-aware dynamic sequential patterns with a stacked convolutional recurrent encoder; c) aggregating the multi-level temporal representations with a recalibration gating mechanism; d) capturing implicit category dependencies with a context-graph neural network.

\subsection{Multi-Scale Pyramid Modulation Network}

We propose a multi-scale pyramid modulation network to capture the multi-grained temporal hierarchical structures of online purchase patterns. Following the pyramid architecture, it consists of bottom-up modulation network and top down modulation network.

The bottom-up network is a hierarchical feedforwad convolutional framework that models temporal feature dynamics of user's online purchase patterns with multi-time granularity information. Each convolutional layer is able to generate high-level semantic representations of purchase patterns by increasing the receptive field of subsequent layers. In particular, given a user $u_{m}$, the bottom-up convolutional network takes the purchase matrix $\mathcal{X}^{m} \in \mathbb{R}^{N \times T}$ as input and perform convolutional operations on $L$ levels (indexed by $l$ ) of time resolutions to generate feature representations $\mathbf{Y}_{m}^{l} \in \mathbb{R}^{N \times \frac{T}{2^{l} \times e_{l}}}$ in $l$-th level ( $e_{l}$ denotes the channel size of $l$-th layer), each of which operate at increasing temporal resolution. Formally, each convolutional layer can be represented as follows:

$$
\mathbf{Y}_{m}^{l}=\operatorname{ReLU}\left(\mathbf{W}_{b o m}^{l} * \mathbf{Y}_{m}^{(l-1)}+\mathbf{b}_{b o m}^{l}\right)
$$

where, $*$ is the convolutional operation, $\mathbf{W}_{b o m}^{l}$ and $\mathbf{b}_{b o m}^{l}$ represents the transformation matrix and bias term in $l$-th layer of the bottom-up network, respectively. We output the feature representation on $X^{m}$ of user $u_{m}$ with highest level semantic signals of at the final layer, i.e., $\mathbf{Y}_{m}^{L}$.
In the bottom-up network, we set the kernel size as $3 \times 1$ at each layer with a stride of 2 . The number of channels in our bottom-up network is set as $(4,16,32,64)$ for successive convolutional layers. After stacking $L$ convolutional layers, the bottom-up network will generate intermediate latent representations (i.e., $\mathbf{Y}_{m}^{L}$ ) of user $u_{m}$ 's purchase behavior on all categories from previous $T$ time steps. During the process of feature representation learning, to avoid the aliasing effect of sampling across dimensions with different categories [26, 32], we only perform convolutional operations on the temporal dimension and fix the category dimension in the input matrix of each layer.

The feature maps generated from the bottom-up network encode the temporal patterns from low to high granularities. However, different levels are expected to be characterized with different granularities of temporal patterns. The flexibility of the mere bottom-up network is limited in the sense that the granularities of the temporal patterns captured in the feature maps at two consecutive layers are highly sensitive to hyperparameter settings (i.e.kernel size, stride size). To address this issue, we leverage a top-down modulation network, a symmetric structure of bottum-up network with a reverse order from low to high granularities, while utilizing lateral connections to enhance the mapping operation between the original layer and the corresponding reconstructed layer.

In our multi-scale pyramid modulation architecture, the topdown modulation network starts from the last layer of the above bottom-up feedforward network. Then, we apply the stacked convolutional layers to transmit higher-level semantic signals into the feature representation learning process of purchase patterns with lower-level resolutions. More specifically, we use convolutional neural network with upsampling operations to propagate encoded feature representations into each $l$-th layer $(1 \leq l \leq L)$ in the top-down architecture. The formulation of feature representation $\mathbf{Z}_{m}^{l} \in \mathbb{R}^{N \times \frac{T}{2^{l}} \times g_{l}}$ ( $g_{l}$ is the channel dimension in top-down network) for $l$-th layer can be given as:

$$
\mathbf{Z}_{m}^{l}=\operatorname{ReLU}\left(\mathbf{W}_{\text {top }}^{l} * \mathbf{Z}_{m}^{(l+1)}+\mathbf{b}_{\text {top }}^{l}\right)
$$

where $\mathbf{W}_{\text {top }}^{l}$ and $\mathbf{b}_{\text {top }}^{l}$ are learned parameters. Furthermore, we enhance the feature learning of top-down framework with lateral connections between the bottom-up and top-down networks, which incorporates the resolution contextual signals to guide the feature learning of top-down network. Particularly, first, the lateral connection leverages point-wise convolutional neural networks to transform $\left(\frac{T}{2^{l}} \times N \times e_{l}\right)$ from bottom-up architecture to $\left(\frac{T}{2^{l}} \times N \times g_{l}\right)$, where $g_{l}$ is the number of channels in top-down network. Then, the element-wise addition is applied to the same level of bottom-up and top-down layers. Therefore, the learned feature representations are flexible and comprehensive in the sense that the representations capture a various range of temporal pattern granularities.

\subsection{Multi-Modal Conv-Recurrent Encoder}

Given the generated feature representations $\left\{\mathbf{Z}_{m}^{1}, \ldots, \mathbf{Z}_{m}^{l}, \ldots, \mathbf{Z}_{m}^{L}\right\}$ from the above pyramid architecture, we develop a multi-modal convolutional recurrent encoder to model multiple temporal dynamics of purchase patterns across time steps. The Conv-RNN model has been introduced as a RNN variant for sequence modeling with both spatial and temporal information [31]. Our Conv-RNN cell involves 

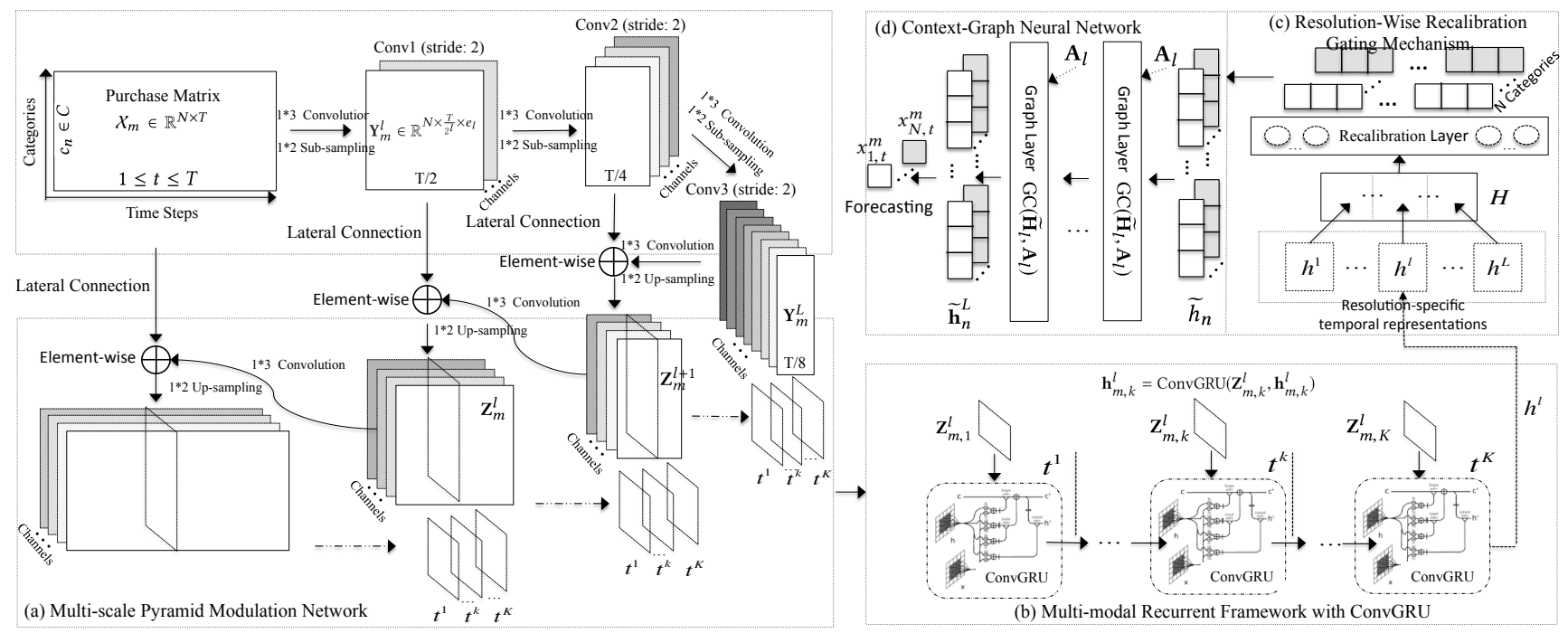

Figure 2: The Graph Multi-Scale Pyramid Networks (GMP) Framework. (a) The lateral connection leverages point-wise convolutional neural networks to transform feature representations (i.e., $\left(\frac{T}{2^{l}} \times N \times e_{l}\right)$ to $\left.\left(\frac{T}{2^{l}} \times N \times g_{l}\right)\right)$ between bottom-up and top-down pathways. $\bigoplus$ represents the element-wise addition operation. (c) Each resolution-specific feature representation $Z_{m}^{l}$ from $l$-th level of pyramid architecture will be fed into a ConvGRU encoder. We plot only one level of multi-modal recurrent framework, due to space limit. (d) $\mathbf{H} \in \mathbb{R}^{N \times\left(L \cdot d_{c}\right)}$ is a multi-resolution representation matrix which is concatenated from the encoded resolution-specific temporal representations $\mathbf{h}^{l}(1 \leq l \leq L)$.

convolutional operations with GRU unit, which can be formally presented as:

$$
\begin{aligned}
r_{k} & =\sigma\left(\mathbf{W}_{x r} * X_{k}+\mathbf{W}_{h r} * h_{k-1}+b_{r}\right) \\
z_{k} & =\sigma\left(\mathbf{W}_{x z} * X_{k}+\mathbf{W}_{h z} * h_{k-1}+b_{z}\right) \\
\mathbf{J}_{k} & =r_{k} \circ J_{k-1}+z_{k} \circ \tanh \left(\mathbf{W}_{x c} *+\mathbf{W}_{h c} * h_{k-1}+b_{c}\right) \\
\mathbf{h}_{k} & =z_{k} \circ \tanh \left(\mathbf{J}_{k}\right)
\end{aligned}
$$

where $r_{k}$ and $z_{k}$ represents the outputs of reset gate and update gate at the $k$-th time step in recurrent neural network. $*$ is the convolutional operation. We denote the cell output at the $k$-th time step as $J_{k}$ and the hidden state of a cell at the $k$-th time step as $\mathbf{h}_{k}$.

To model time-evolving temporal dependencies of multi-level feature representations from our pyramid modulation network, we develop a convolutional recurrent encoder, to account for each resolution-specific feature representation $\mathbf{Z}_{m}^{l}$ of user $u_{m}$. In particular, we first slice each learned feature representations $\mathbf{Z}_{m}^{l}$ over time dimension and then obtain $\left\{\mathbf{Z}_{m, 1}^{l} \ldots, \mathbf{Z}_{m, k}^{l}, \ldots, \mathbf{Z}_{m, \frac{T}{2} l}^{l}\right\}$, where $\mathbf{Z}_{m, k}^{l} \in \mathbb{R}^{N \times g^{l}}$. Then, we feed the last $\mathrm{K}$ data points of each level into its corresponding ConvGRU layer to encode sequential patterns. Formally, we denote the derivations of hidden vector representation $\mathbf{h}_{k}^{l} \in \mathbb{R}^{N \times d_{c}}$ for $l$-th pyramid scale as $\mathbf{h}_{k}^{l}=$ $\operatorname{ConvGRU}\left(\mathbf{Z}_{m, k}^{l}, \mathbf{h}_{k-1}^{l}\right)$.

The advantages of utilizing ConvGRU lie in: (i) it is able to capture temporal correlations across time steps; (ii) the convolution operator enables the consideration of topological information in $\mathbf{Z}_{m, k}^{l}$. An alternative way to consider temporal dependencies is to directly flatten the input matrix $\mathbf{Z}_{m, k}^{l}$ into a vector and then feed

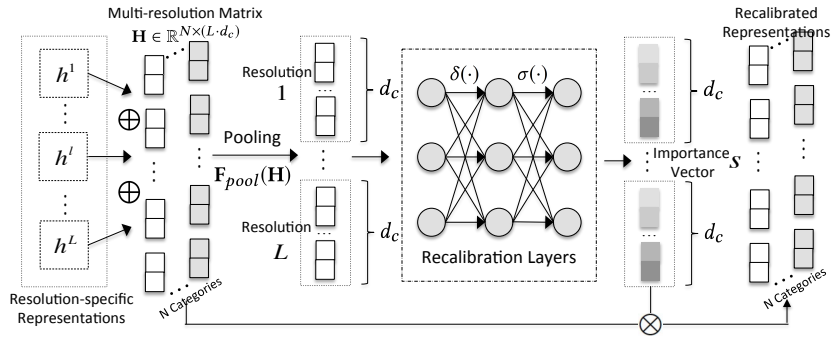

Figure 3: Illustration of the recalibration gating mechanism.

into a GRU layer. However, by doing so, it is likely that a lot of contextual spatial signals would be lost.

\subsection{Resolution-Wise Gating Mechanism}

In our GMP framework, the goal of the resolution-wise recalibration gating mechanism is to select the most informative components from the encoded resolution-specific temporal representations $\mathbf{h}^{l}(1 \leq l \leq L)$, and then aggregate the representation of informative resolution elements to characterize user's purchase patterns. In order to exploit the element dependencies over resolution dimension, we first concatenate $\mathbf{h}^{l}$ from each $l$-th layer to construct a multi-resolution representation matrix $\mathbf{H} \in \mathbb{R}^{N \times\left(L \cdot d_{c}\right)}$ as:

$$
\mathbf{H}=\mathbf{h}^{1} \oplus \ldots \oplus \mathbf{h}^{l} \oplus \ldots \oplus \mathbf{h}^{L}
$$

where $\oplus$ represents the concatenation operation. Then, we apply global average pooling $F_{\text {pool }}$ on $\mathbf{H}$ over $N$ category dimensions to 
produce the summary of each element-wise $\left(\left[1, \ldots, p, \ldots,\left(L \cdot d_{c}\right)\right]\right.$ indexed by $p$ ) representations as:

$$
\varphi^{(p)}=\mathbf{F}_{p o o l}(\mathbf{H})=\frac{1}{N} \sum_{n=1}^{N} H^{(p)}
$$

where $\varphi^{(p)}$ denotes the $p$-th element in the pooling summarized vector $\varphi \in \mathbb{R}^{1 \times\left(L \cdot d_{c}\right)}$. We then propose a resolution-aware recalibration gating mechanism to recalibrate the information distribution among all fine-grained elements across resolutions, i.e., $1 \leq q \leq\left(L \cdot d_{c}\right)$. We define $s$ as the importance score vector to indicate the importance of all elements in $\mathbf{H}$. Formally, our gating mechanism can be represented as follows:

$$
s=\sigma\left(\mathbf{W}_{2} \cdot \delta\left(\mathbf{W}_{1} \cdot \varphi\right)\right)
$$

Here, $\mathbf{W}_{1}$ and $\mathbf{W}_{2}$ is the corresponding transformation matrix of two fully connected neural network layers. $\sigma$ and $\delta$ dentes the Sigmoid and ReLU activation function, respectively. Finally, the aggregation process is given as follows:

$$
\widetilde{h}_{n}=h_{n, 1} \circ s_{1} \uplus \ldots \uplus h_{n, l} \circ s_{l} \uplus \ldots \uplus h_{n, L} \circ s_{L} ; l \in[1, \ldots, L]
$$

where $\widetilde{h}_{n}$ denotes the summarized multi-resolution representation on category $c_{n}$ and $s_{l}$ is the sub-vector of $s$ corresponding to the $l$-th resolution. $\uplus$ and $\circ$ is defined as the element-wise summation and multiplication, respectively. $s^{(p)}$ is the $p$-th entry of vector $s$.

\subsection{Context-Graph Neural Network}

In this subsection, we show how to encode the contextual signals in our predictive solution GMP, by modeling the inter-dependencies of purchase behavior between different categories with graph neural network. We first define the following input.

Definition 2. Category Graph G. Our context-graph neural network is defined over the category graph $G=(V, E)$, where $V$ and $E$ is the set of all vertices and edges, respectively. Each vertex corresponds to a specific category $c_{n}$ and each edge indicates the relationship between two product categories.

Definition 3. Adjacent Matrix $A . A \in \mathbb{R}^{N \times N}$ represents the adjacency matrix whose entries (i.e., $a_{n, n^{\prime}}$ ) is the correlations between two categories (i.e., $c_{n}$ and $c_{n^{\prime}}$ ). In particular, $a_{n, n^{\prime}}$ is the estimated similarity between the learned summarized representation vector $\widetilde{h}_{n}$ and $\widetilde{h}_{n^{\prime}}$ (encoded from the recalibration gating mechanism), i.e., $a_{n, n^{\prime}}^{l}=\exp ^{-d\left(\widetilde{\boldsymbol{h}}_{n, l}, \widetilde{\boldsymbol{h}}_{n^{\prime}, l}\right)}$, where $d(\cdot)$ represents the Euclidean distance [17] between $\widetilde{\boldsymbol{h}}_{n, l}$ and $\widetilde{\boldsymbol{h}}_{n^{\prime}, l}$.

A graph convolution layer GC( $\cdot)$ receives an input $\widetilde{\mathbf{H}}_{l} \in \mathbb{R}^{N \times d_{l}}$ from $l$-th layer and produces $\widetilde{\mathbf{H}}_{l} \in \mathbb{R}^{N \times d_{l+1}}$ as:

$$
\widetilde{\mathbf{H}}_{l+1}^{*}=\operatorname{GC}\left(\widetilde{\mathbf{H}}_{l}, \mathbf{A}_{l}\right)=\delta\left(\mathbf{A}_{l} \widetilde{\mathbf{H}}_{l} \mathbf{W}_{g}\right)
$$

where $\mathbf{W}_{g} \in \mathbb{R}^{d_{l} \times d_{l+1}}$ denotes the feature transformation matrix. $\delta(\cdot)$ is a point-wise non-linearity ReLU activation function. In each $l$-th graph convolution layer, the adjacency matrix $\mathbf{A}_{l}$ is updated based on the new estimated vertex embedding vectors $\widetilde{h}_{n}(n \in$ $[1, \ldots, N])$. We further normalize the trainable adjacency matrix $\mathbf{A}$ with a stochastic kernel using a softmax along each row.

In our framework, we set the depth of our context-graph convolution network as the order of the graph diameter, so that all

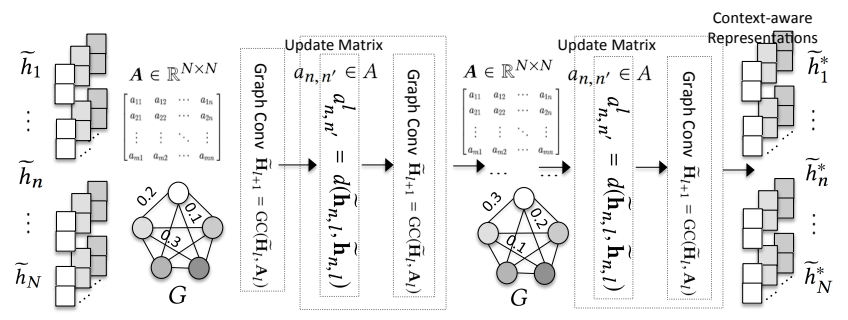

Figure 4: Illustration of the context-graph neural network.

vertex contextual information from the the entire graph $G$ could be incorporated in the learned vertex representation vectors. Instead of handcrafed category graph construction, our context-graph neural network enables the automatic discovery of meaningful and useful dependency features from category representations. The probability of user $u_{m}$ purchase items of $n$-th category at $t$-th time step is computed by feeding each fused embedding vector to the logistic regression.

\subsection{Model Inference}

In general, our online purchase prediction can be regarded as a classification problem. We utilize cross entropy as the metric in our loss function which is defined as follows:

$$
\mathscr{L}(\Theta)=-\sum_{(m, n, t) \in D} x_{n, t}^{m} \log \hat{x}_{n, t}^{m}+\left(1-x_{n, t}^{m}\right) \log \left(1-\hat{x}_{n, t}^{m}\right)
$$

where $\Theta$ represents all learnable parameters in GMP. $\hat{x}_{n, t}^{m}$ denotes the estimated probability of user $u_{m}$ purchase items of $n$-th category at $t$-th time step. Here, $D$ denotes the set of observed purchase interactions in the training process. During the training phase, we use Adam optimizer to infer model parameters by minimizing the loss function. In addition, we apply Batch Normalization [4] to reduce the internal covariance shift by transforming the input to zero mean/unit variance distributions in each mini-batch training.

\section{EVALUATION}

To comprehensively evaluate our proposed model, we perform experiments to answer the following questions:

- Q1: How is the overall prediction performance of our GMP as compared with various types of state-of-the-art methods across different categories?

- Q2: How does GMP perform compared with competitive approaches in forecasting individual category-specific purchases?

- Q3: How is the ranking-based performance of GMP in forecasting users' category-specific purchases?

- Q4: How do the different components (e.g., resolution-wise recalibration gating mechanism and context-graph neural network) of GMP contribute to the model performance?

- Q5: How does our GMP model work with different evaluated time resolution (e.g., 1 day, 3days, and etc)?

- Q6: How do different hyperparameter settings affect the performance of GMP (Refer to Appendix Section 7 for details)? 
Table 1: Statistics of the experimented datasets.

\begin{tabular}{|c|c|c|c|}
\hline Dataset & \# of Users & \# of Categories & \# of Purchases \\
\hline JD-1 & 3,362 & 12 & 470,634 \\
\hline JD-2 & 11,593 & 12 & $2,127,351$ \\
\hline JD-3 & 59,476 & 12 & $3,377,133$ \\
\hline
\end{tabular}

- Q7: How is the interpretation of our GMP framework in capturing dynamic category dependencies?

\subsection{Experimental Settings}

4.1.1 Datasets. We experimented with three real-world online purchase datasets collected from JD.com (JD-1, JD-2 and JD-3) within the time period from $01 / 01 / 2015$ to $06 / 30 / 2018$. Each online purchase record is in the format of (product category, user id, timestamp). These datasets were collected with different data scales (i.e., the number of users and purchases), reflective of different degrees of online purchase activities. Table 1 summarizes the statistics about our experimented datasets. The details of data partition for training, validation, and testing are presented in Appendix Section 7.

4.1.2 Methods for Comparison. We compared it with the following state-of-the-art methods from various research lines:

Recurrent Neural Network-based Methods: Since RNNs have shown their superiority in handling time-ordered sequential data as compared to conventional time series analysis methods (e.g., ARIMA), we considered two developed variants of RNNs in the performance evaluation.

- Sequential Prediction with Recurrent Neural Network (SPRNN) [36]: a deep learning approach which models the dependency on user's online behavior prediction via the recurrent neural network structure.

- Stacked Long Short-Term Memory Model (ST-LSTM) [33]: ST-LSTM is a mixture deep recurrent architecture on dynamic time series data by unifying stacked LSTM networks.

Attentive Recurrent Models: we further compare with another line of methods that models time-stamped data with the integration of attention mechanism and recurrent neural networks.

- Dual-Stage Attentive Recurrent Networks (DA-RNN) [19]: DA-RNN is a dual stage attentive time series prediction method which consists of an encoder with an input attention mechanism and a decoder with a temporal attention mechanism.

- Attentive Bidirectional Recurrent Model (Dipole) [16]: It models the temporal and high dimensional time series data by employing bidirectional recurrent neural networks and further interpreting learned representations with attention mechanisms.

Recommendations Techniques with Temporal Dynamics: In the performance comparison, we also include recommendation models which care about temporal drift effect.

- Long- and Short-term Time-Series Network (LSTNet) [10]: LSTNet combines the convolution neural network and the recurrent neural network, to extract short-term local dependency patterns and discover long-term patterns for time series trends.

- Conv-Sequence Recommendation Model (Caser) [22]: Caser first embeds a sequence of recent items into a tensor and then learns sequential patterns using convolutional filters.
Table 2: Prediction results across different categories in terms of Macro-F1 (Mac-F1) and Micro-F1 (Mic-F1).

\begin{tabular}{l|c|c|c|c|c|c}
\hline Dataset & \multicolumn{2}{|c|}{ JD-1 } & \multicolumn{2}{c|}{ JD-2 } & \multicolumn{2}{c}{ JD-3 } \\
\hline Metrics & Mac-F1 & Mic-F1 & Mac-F1 & Mic-F1 & Mac-F1 & Mic-F1 \\
\hline Caser & 0.1994 & 0.1303 & 0.1969 & 0.1232 & 0.1423 & 0.0903 \\
Dipole & 0.2237 & 0.1490 & 0.1720 & 0.0626 & 0.1290 & 0.0634 \\
LSTNet & 0.2638 & 0.0784 & 0.3332 & 0.1325 & 0.0829 & 0.0393 \\
SP-RNN & 0.2100 & 0.1382 & 0.3050 & 0.1921 & 0.1467 & 0.0951 \\
DARNN & 0.2948 & 0.1537 & 0.1720 & 0.0626 & 0.0829 & 0.0393 \\
ST-LSTM & 0.2736 & 0.1823 & 0.208 & 0.1262 & 0.1514 & 0.0970 \\
mWDN & 0.3426 & 0.2270 & 0.2685 & 0.1717 & 0.1709 & 0.1638 \\
\hline GMP & $\mathbf{0 . 3 7 9 9}$ & $\mathbf{0 . 2 4 4 4}$ & $\mathbf{0 . 3 5 3 1}$ & $\mathbf{0 . 2 2 3 2}$ & $\mathbf{0 . 3 1 1 8}$ & $\mathbf{0 . 1 9 1 9}$ \\
\hline
\end{tabular}

Hybrid Multi-level Model: Finally, we compare our GMP with a hybrid time series analysis approach with multi-level wavelet decomposition networks.

- Multilevel Wavelet Decomposition Network (mWDN) [24]: mWDN is a wavelet-based neural network architecture which integrates the multilevel discrete wavelet decomposition and the frequency-aware LSTM for time series forecasting.

4.1.3 Evaluation Protocols. To fully measure the effectiveness of our GMP framework for online purchase prediction, we adopt three types of evaluation metrics:

- Prediction across Categories. We use Marco-F1 and Micro-F1 to evaluate the prediction accuracy across different product categories [13]. They indicate the overall performance across different classes (categories).

- Prediction on Individual Categories. We use F1-score (harmonic mean to balance precision and recall) and Area Under Curve (AUC) [3] as evaluation metrics for the accuracy of predicting user's purchases on each individual category.

- Ranking-based Performance. To assess the ranked list with the ground-truth user who has actual category-specific purchases, we adopt Mean Average Precision (MAP)@k and Normalized Discounted Cumulative Gain (NDCG)@k [5], where MAP@k computes the average precision of top- $k$ ranked users and NDCG@k accounts for the position of hit for specific $n$-th category.

Note that all metrics are the higher the better.

4.1.4 Parameter Settings. The parameter settings and implementation details are presented in Appendix (Section 7).

\subsection{Overall Performance Comparison (Q1)}

Table 2 shows both the forecasting accuracy across categories of different methods on three datasets in terms of Macro-F1 and MicroF1. We summary key observations as follows:

(1) GMP achieves the best performance and obtains high improvements over different types of state-of-the-art methods in all cases. This sheds lights on the benefit of our GMP model which jointly captures multi-scale temporal dynamics and arbitrary category dependencies. The performance is followed by mWDN which decomposes an online purchase series into a group of sub-series to capture multi-dimensional frequency factors. This further verifies the utility of considering multi-dimensional temporal information 
Table 3: Purchase prediction results on individual categories in terms of F1-score and AUC.

\begin{tabular}{|c|c|c|c|c|c|c|c|c|c|c|}
\hline Category & Density & Metrics & Caser & Dipole & LSTNet & SP-RNN & DARNN & ST-LSTM & mWDN & GMP \\
\hline \multirow{2}{*}{ Beauty \& Care } & \multirow{2}{*}{$d s=2.9 \%$} & F1-score & 0.0440 & 0.0704 & 0.0584 & 0.0590 & 0.0295 & 0.1173 & 0.1899 & 0.2439 \\
\hline & & AUC & 0.7311 & 0.7424 & .7574 & .7284 & .7433 & 0.7555 & 0.7646 & 0.7832 \\
\hline \multirow{2}{*}{ Clothing \& Sho } & \multirow{2}{*}{$d s=3.83 \%$} & F1-score & 0.2396 & 0.2592 & 0.2607 & 0.2291 & 0.2736 & 0.1505 & 0.2201 & 0.2942 \\
\hline & & AUC & 0.7049 & 0.7357 & 0.7308 & 0.7181 & 0.7227 & 0.7490 & 0.7490 & 0.8212 \\
\hline \multirow{2}{*}{ Computers \& Office } & \multirow{2}{*}{$d s=1.25 \%$} & F1-score & 0.1820 & 0.1935 & 0.1874 & 0.1790 & 0.1548 & 0.2283 & 0.2050 & 0.2807 \\
\hline & & AUC & 0.6888 & 0.7007 & 0.7052 & 0.6904 & 0.6729 & 0.7049 & 0.6984 & 0.7420 \\
\hline \multirow{2}{*}{ Electronics } & \multirow{2}{*}{$d s=3.16 \%$} & F1-score & 0.3189 & 0.3418 & 0.3461 & 0.3277 & 0.3279 & 0.3755 & 0.1302 & 0.4307 \\
\hline & & AUC & 0.7562 & 0.7745 & 0.7464 & 0.7603 & 0.7551 & 0.7855 & 0.7901 & 0.8233 \\
\hline \multirow{2}{*}{ Food \& Grocery } & \multirow{2}{*}{$d s=3.65 \%$} & F1-score & 0.3511 & 0.3891 & 0.5146 & 0.3684 & 0.4959 & 0.4512 & 0.5743 & 0.5884 \\
\hline & & AUC & 0.6949 & 0.7008 & 0.7227 & 0.6959 & 0.7101 & 0.7022 & 0.6968 & 0.7370 \\
\hline \multirow{2}{*}{ Fresh Food } & \multirow{2}{*}{$d s=1.85 \%$} & F1-score & 0.0997 & 0.1274 & 0.0896 & 0.1131 & 0.0689 & 0.1587 & 0.1361 & 0.1516 \\
\hline & & AUC & 0.6305 & 0.6484 & 0.6603 & 0.6352 & 0.6365 & 0.6607 & 0.6591 & 0.6832 \\
\hline \multirow{2}{*}{ Health \& Medicine } & \multirow{2}{*}{$d s=1.61 \%$} & F1-score & 0.1010 & 0.1088 & 0.1804 & 0.1077 & 0.1652 & 0.1227 & 0.1191 & 0.1270 \\
\hline & & AUC & 0.6894 & 0.7151 & 0.7328 & 0.6983 & 0.7267 & 0.7307 & 0.7383 & 0.7788 \\
\hline \multirow{2}{*}{ Home \& Furniture } & \multirow{2}{*}{$d s=1.97 \%$} & F1-score & 0.1847 & 0.2321 & 0.3442 & 0.2037 & 0.3804 & 0.3267 & 0.2301 & 0.4045 \\
\hline & & AUC & 0.7380 & 0.7563 & 0.7712 & 0.7418 & 0.7610 & 0.7626 & 0.7672 & 0.7956 \\
\hline \multirow{2}{*}{ Luggage \& G } & \multirow[b]{2}{*}{$\%$} & F1-score & 0.1896 & 0.2102 & 0.2546 & 0.1998 & 0.3204 & 0.2268 & 0.1645 & 0.3440 \\
\hline & & AUC & 0.6619 & 0.6812 & 0.6864 & 0.6695 & 0.6874 & 0.6893 & 0.6832 & 0.7249 \\
\hline \multirow{2}{*}{ Mother \& Baby } & \multirow{2}{*}{$d s=2.11 \%$} & F1-score & 0.1430 & 0.1447 & 0.1002 & 0.1361 & 0.1100 & 0.1452 & 0.1159 & 0.1515 \\
\hline & & AUC & 0.6108 & 0.6431 & 0.6520 & 0.6241 & 0.6469 & 0.6553 & 0.6542 & 0.7190 \\
\hline \multirow{2}{*}{ Travel \& Outdoors } & \multirow{2}{*}{$d s=1.38 \%$} & F1-score & 0.0436 & 0.0617 & 0.1382 & 0.0566 & 0.1562 & 0.0892 & 0.1957 & 0.2611 \\
\hline & & AUC & 0.7197 & 0.7412 & 0.7603 & 0.7207 & 0.7571 & 0.7548 & 0.7682 & 0.8016 \\
\hline \multirow{2}{*}{ Toys \& Instruments } & \multirow{2}{*}{$d s=$} & F1-score & 0.1179 & 0.1684 & 0.2426 & 0.1422 & 0.1274 & 0.2062 & 0.2790 & 0.3172 \\
\hline & & AUC & 0.6487 & 0.6613 & 0.6975 & 0.6545 & 0.6771 & 0.6750 & 0.6680 & 0.7266 \\
\hline
\end{tabular}

in predicting online purchases. However, mWDN fails to consider high-order inter-dependencies across time resolutions. In contrast, GMP dynamically learning cross-level semantics from data, which shows remarkably flexibility and superiority.

(2) With the increase of the number of users and purchases, the performance improvement of GMP compared with other baselines also increases. The reason may be that the learned feature representations are more informative for a relatively larger scale of purchase activities, in which the multi-scale dynamics of online purchase behavior is more obvious. In addition, there is no obvious winner among attentive recurrent models and temporal recommendation techniques. This again confirms that only considering data dependencies and interactions from singular temporal dimension is insufficient to model complex sequential purchase transition regularities exhibited with multi-scale behavior dynamics.

\subsection{Category-Specific Prediction Accuracy (Q2)}

We investigated the effectiveness of GMP in predicting users' purchases on each individual category and report the evaluation results in Table 3. We can observe that our GMP achieves the best performance in all forecasting cases. Additionally, we could notice that obvious improvements can also be obtained by GMP in predicting purchases of different categories, which shows the robustness of our GMP's prediction performance.

\subsection{Ranking Performance Comparison (Q3)}

Furthermore, we also measure the ranking quality of top- $k$ predicted users for future category-specific purchase of our GMP method with varying $k$ from 200 to 1000 . The evaluation results (measured by MAP@k and NDCG@k) of GMP and the best performed baseline
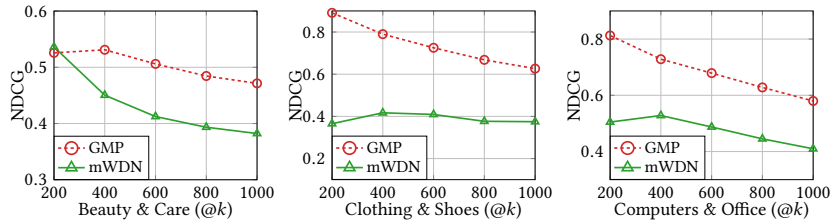

Figure 5: Purchase prediction results on individual categories in terms of NDCG@k.
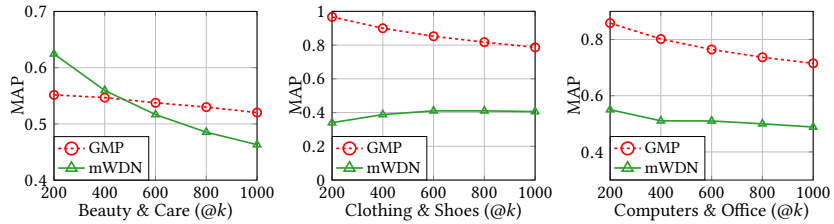

Figure 6: Purchase prediction results on individual categories in terms of MAP@k.

$(\mathrm{mWDN})$ on JD-1 data traces are presented in Figure 6 and Figure 5. We can observe that GMP achieves the best performance under different values of $k$, which suggests that our GMP model assigns higher score to the true users in the top- $k$ ranked list and hit the ground truth at top positions.

\subsection{Component-Wise Evaluation of GMP (Q4)}

In our evaluation, we consider five variants of the proposed method corresponding to different analytical aspects:

- Effect of Category Dependencies $G M P$-g: A simplified version of GMP which does not include context-graph neural module to consider dependencies among categories. 


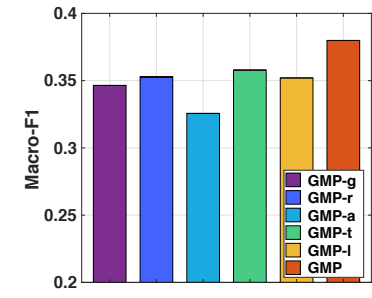

(a) Macro-F1

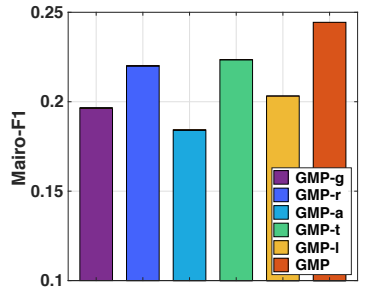

(b) Micro-F1
Figure 7: Evaluation on GMP variants.

- Effect of Recalibration Gating Mechanism. GMP-r: A simplified version of GMP which does not include the recalibration gating mechanism to model element-wise correlations from encoded resolution-aware representations.

- Aliasing Effect in Pyramid Modulation Network. GMP-a: During the process of feature map generation in the bottom-up convolutional networks, we perform the convolutional operation with $3 \times 3$ filter size to directly consider category correlations using the local structure information.

- Impact of Modeling Cross-level Semantics. GMP-t: We only reply on the bottom-up convolutional network to encode the underlying multi-dimensional structures of evolving temporal dependencies, i.e., without the top-down modulation network in the multi-scale pyramid architecture.

- Effectiveness of Encoded Multi-Scale Temporal Dynamics. $G M P-1$ : To further evaluate the quality of the latent representations learned from our designed multi-scale pyramid networks, we first perform flatten operation on the learned resolutionspecific temporal representations and then feed them into an integrative framework of LSTM and MLP for making predictions.

We report the evaluation results in Figure 7. We can notice that the full version of our developed framework GMP achieves the best performance in all cases, which suggests: (i) the effectiveness of GMP in capturing the category-aware inter-relations between users' online purchases in a dynamic environment; (ii) the efficacy of the designed recalibration gating mechanism in handling hierarchical structural relations among resolution-aware purchase patterns; (iii) the rationality of GMP in addressing the aliasing effect in the feature representation process from different modalities; (iv) the effectiveness of the top-down modulation networks for helping GMP transmit high-level temporal semantics back to low-level latent representations.

\subsection{Effect of Evaluated Time Resolution (Q5)}

To further investigate the robustness of GMP, we evaluate the model performance with different resolutions of evaluated time step (i.e., from the finest resolution-1 day to the coarsest resolution-10 days) as shown in Figure 8. We can observe that GMP consistently outperforms the best performed baseline mWDN with respect to different evaluated resolutions for all category cases, which demonstrates the robustness of our GMP framework in various purchase prediction scenarios with different granularity of target time period.

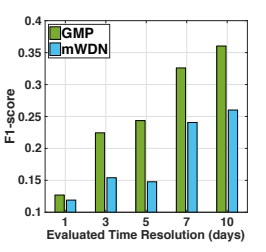

(a) Health \& Medicine

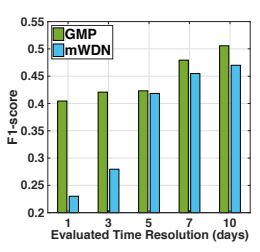

(b) Home \& Furniture

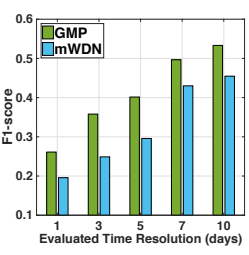

(c) Travel \& Outdoors
Figure 8: Prediction results on individual categories v.s. time resolution of target period.
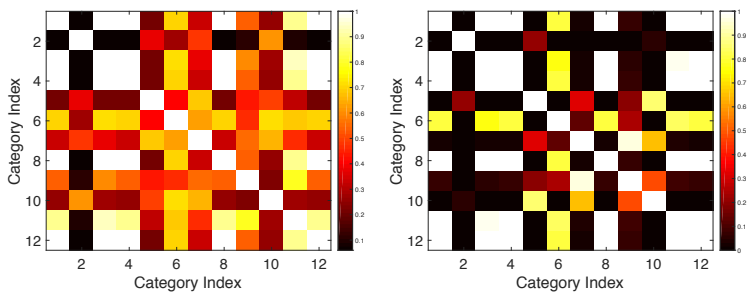

(a) Adjacent Matrix $A$ from $1^{\text {st }}$ Layer (b) Adjacent Matrix $A$ from $2^{\text {nd }}$ Layer

Figure 9: The adjacent matrix $A$ across different categories updated from the first and second layer in our GMP. Category index is consistent with the order in Table 3.

\subsection{Case Study (Q7)}

Apart from the superior forecasting performance, another key advantage of GMP is its ability in interpreting the dependencies across different categories in predicting online purchases. To demonstrate this, we perform case studies to show the explainability of our framework by visualizing the dynamic adjacent matrix $A$ (dependency weights between categories) as shown in Figure 9. GMP will update the adjacent matrix $A$ through each layer. We could observe that GMP enables the dynamic modeling of dynamic and arbitrary correlations between different categories.

\section{RELATED WORK}

Neural Next-Item Recommendation. Many deep neural network models for next-item recommendation seek to model itemitem transition among successive items [9, 12, 35]. For instance, Yuan et al. [35] studied the next-item recommendation problem by considering both short- and long-range item dependencies. Kang et al. [9] proposed a self-attention based sequential model to make next item predictions based on relatively few items, to model user's sequential patterns. Complementary to next-item recommendations, accurate prediction of online purchase activities can help sequential recommender systems to generate more relevant items for ranking and offer more effective personalized recommendations.

Time Series Data Forecasting. Although conventional approaches (e.g., (ARIMA) [18] and Support Vector Regression (SVR) [21]) work well for time series analysis in a static scenario, they are less applicable to capture the dynamic patterns among time series data. To address this problem, RNNs-based methods (e.g., LSTM [29] and GRU [8]) were proposed and have shown in their success in modeling sequential data. Additionally, further attempt-the integration 
of CNN-RNN network structure and attention mechanisms-was made to adaptively identify values relevant from relevant time steps for making forecasting $[7,19,24]$. However, most of the existing deep neural network methods failed to pay attention to multi-levels of temporal dynamics in the time series of user purchases, which is the key concern of this work.

Time-stamped Behavior Modeling. There exists a good amount of research work in modeling various behavioral time-stamped data $[1,11,14,20,30]$. For example, Lian et al. [14] aimed to explore user web activities with the consideration of high-order feature interactions. Qiu et al. [20] investigated user social activities using their local network information. Online purchases are different from the above time-stamped human behavior. The sequential transition regularities of purchase patterns is often exhibited with both highordered time-dependent and temporal hierarchy nature, which pose difficulties to comprehensively explore costumer purchases with a multi-dimensional structure.

\section{CONCLUSION}

This work contributes a new framework, named GMP for online purchase prediction via modeling behavior dynamics from both multi-scale temporal patterns and arbitrary category dependencies. Particularly, we develop a multi-scale pyramid neural network architecture to explore the high-order correlations underlying multiresolution online purchase patterns. In addition, we propose a recalibration gating mechanism that is tailored to cooperate with a hierarchical recurrent framework for multi-resolution pattern fusion. With the help of context-graph neural network module, we further consider contextual signals in encoding complex relations between category-specific purchases. Finally, we perform extensive experiments on three real-world datasets. Evaluation results shown that the proposed model significantly outperforms the state-of-theart methods.

\section{ACKNOWLEDGMENTS}

This work is supported in part by the National Science Foundation under Grant No. IIS-1447795.

\section{REFERENCES}

[1] Bokai Cao, Lei Zheng, Chenwei Zhang, Philip S Yu, Andrea Piscitello, John Zulueta, Olu Ajilore, Kelly Ryan, and Alex D Leow. 2017. DeepMood: modeling mobile phone typing dynamics for mood detection. In KDD. ACM, 747-755.

[2] Shiyu Chang, Yang Zhang, Jiliang Tang, Dawei Yin, Yi Chang, Mark A HasegawaJohnson, and Thomas S Huang. 2017. Streaming recommender systems. In $W W W$. International World Wide Web Conferences Steering Committee, 381-389.

[3] Yuxiao Dong, Jing Zhang, Jie Tang, Nitesh V Chawla, and Bai Wang. 2015. Coupledlp: Link prediction in coupled networks. In KDD. ACM, 199-208.

[4] Xiangnan He and Tat-Seng Chua. 2017. Neural factorization machines for sparse predictive analytics. In SIGIR. ACM, 355-364.

[5] Binbin Hu, Chuan Shi, Wayne Xin Zhao, and Philip S Yu. 2018. Leveraging meta-path based context for top-n recommendation with a neural co-attention model. In KDD. ACM, 1531-1540.

[6] Diane J Hu, Rob Jall, and Josh Attenberg. 2014. Style in the long tail: discovering unique interests with latent variable models in large scale social e-commerce. In KDD. ACM, 1640-1649.

[7] Chao Huang, Chuxu Zhang, Jiashu Zhao, Xian Wu, Dawei Yin, and Nitesh V Chawla. 2019. MiST: A Multiview and Multimodal Spatial-Temporal Learning Framework for Citywide Abnormal Event Forecasting. In WWW.

[8] Chao Huang, Junbo Zhang, Yu Zheng, and Nitesh V Chawla. 2018. DeepCrime: Attentive Hierarchical Recurrent Networks for Crime Prediction. In CIKM. ACM, 1423-1432.
[9] Wang-Cheng Kang and Julian McAuley. 2018. Self-Attentive Sequential Recommendation. In ICDM. IEEE, 197-206.

[10] Guokun Lai, Wei-Cheng Chang, Yiming Yang, and etc. 2018. Modeling long-and short-term temporal patterns with deep neural networks. In SIGIR. ACM, 95-104.

[11] Ruirui Li, Liangda Li, Xian Wu, Yunhong Zhou, and Wei Wang. 2019. Click feedback-aware query recommendation using adversarial examples. In $W W W$.

[12] Zhi Li, Hongke Zhao, Qi Liu, Zhenya Huang, Tao Mei, and Enhong Chen. 2018. Learning from history and present: next-item recommendation via discriminatively exploiting user behaviors. In KDD. ACM, 1734-1743.

[13] Defu Lian, Kai Zheng, Vincent W Zheng, Yong Ge, Longbing Cao, Ivor W Tsang, and Xing Xie. 2018. High-order Proximity Preserving Information Network Hashing. In KDD. ACM, 1744-1753.

[14] Jianxun Lian, Xiaohuan Zhou, Fuzheng Zhang, Zhongxia Chen, Xing Xie, and Guangzhong Sun. 2018. xDeepFM: Combining Explicit and Implicit Feature Interactions for Recommender Systems. In $K D D$.

[15] Marco Lippi, Matteo Bertini, and Paolo Frasconi. 2013. Short-term traffic flow forecasting: An experimental comparison of time-series analysis and supervised learning. TITS 14, 2 (2013), 871-882.

[16] Fenglong Ma, Radha Chitta, Jing Zhou, Quanzeng You, Tong Sun, and Jing Gao. 2017. Dipole: Diagnosis prediction in healthcare via attention-based bidirectional recurrent neural networks. In KDD. ACM, 1903-1911.

[17] Calvin R Maurer, Rensheng Qi, and Vijay Raghavan. 2003. A linear time algorithm for computing exact Euclidean distance transforms of binary images in arbitrary dimensions. TPAMI 25, 2 (2003), 265-270.

[18] Bei Pan, Ugur Demiryurek, and Cyrus Shahabi. 2012. Utilizing real-world transportation data for accurate traffic prediction. In ICDM. IEEE, 595-604.

[19] Yao Qin, Dongjin Song, Haifeng Chen, Wei Cheng, Guofei Jiang, and Garrison Cottrell. 2017. A dual-stage attention-based recurrent neural network for time series prediction. In $I 7 C A I$.

[20] Jiezhong Qiu, Jian Tang, Hao Ma, Yuxiao Dong, and etc. 2018. DeepInf: Social Influence Prediction with Deep Learning. In KDD. ACM, 2110-2119.

[21] Goce Ristanoski, Wei Liu, and James Bailey. 2013. Time series forecasting using distribution enhanced linear regression. In PAKDD. Springer, 484-495.

[22] Jiaxi Tang and Ke Wang. 2018. Personalized top-n sequential recommendation via convolutional sequence embedding. In WSDM. ACM, 565-573.

[23] Mengting Wan, Di Wang, Jie Liu, Paul Bennett, and Julian McAuley. 2018. Representing and Recommending Shopping Baskets with Complementarity, Compatibility and Loyalty. In CIKM. ACM, 1133-1142.

[24] Jingyuan Wang, Ze Wang, and etc. 2018. Multilevel wavelet decomposition network for interpretable time series analysis. In KDD. ACM, 2437-2446.

[25] Zihan Wang, Ziheng Jiang, Zhaochun Ren, Jiliang Tang, and Dawei Yin. 2018. A path-constrained framework for discriminating substitutable and complementary products in e-commerce. In WSDM. ACM, 619-627.

[26] Maurice Weiler, Mario Geiger, and etc. 2018. 3d steerable cnns: Learning rotationally equivariant features in volumetric data. In NIPS. 10402-10413.

[27] Chao-Yuan Wu, Amr Ahmed, Alex Beutel, Alexander J Smola, and How Jing. 2017. Recurrent recommender networks. In WSDM. ACM, 495-503.

[28] Liang Wu, Diane Hu, Liangjie Hong, and etc. 2018. Turning Clicks into Purchases: Revenue Optimization for Product Search in E-Commerce. In SIGIR. ACM.

[29] Xian Wu, Baoxu Shi, Yuxiao Dong, Chao Huang, and Nitesh V Chawla. 2019. Neural Tensor Factorization for Temporal Interaction Learning. In WSDM. ACM, 537-545.

[30] Xian Wu, Baoxu Shi, Yuxiao Dong, Chao Huang, Louis Faust, and Nitesh V Chawla. 2018. Restful: Resolution-aware forecasting of behavioral time series data. In CIKM. ACM, 1073-1082.

[31] Shi Xingjian, Zhourong Chen, and etc. 2015. Convolutional LSTM network: A machine learning approach for precipitation nowcasting. In NIPS. 802-810.

[32] Jianbo Yang, Minh Nhut Nguyen, Phyo Phyo San, Xiaoli Li, and Shonali Krishnaswamy. 2015. Deep Convolutional Neural Networks on Multichannel Time Series for Human Activity Recognition.. In IFCAI, Vol. 15. 3995-4001.

[33] Rose Yu, Yaguang Li, Cyrus Shahabi, Ugur Demiryurek, and Yan Liu. 2017. Deep learning: A generic approach for extreme condition traffic forecasting. In $S D M$. SIAM, 777-785.

[34] Wenhui Yu, Huidi Zhang, Xiangnan He, Xu Chen, Li Xiong, and Zheng Qin. 2018. Aesthetic-based clothing recommendation. In WWW. ACM, 649-658.

[35] Fajie Yuan, Alexandros Karatzoglou, Ioannis Arapakis, Joemon M Jose, and Xiangnan He. 2019. A Simple Convolutional Generative Network for Next Item Recommendation. In WSDM. ACM.

[36] Yuyu Zhang, Hanjun Dai, Chang Xu, Jun Feng, Taifeng Wang, Jiang Bian, Bin Wang, and Tie-Yan Liu. 2014. Sequential Click Prediction for Sponsored Search with Recurrent Neural Networks.. In AAAI, Vol. 14. 1369-1375.

[37] Dawei Zhou, Jingrui He, and etc. 2018. Sparc: Self-paced network representation for few-shot rare category characterization. In KDD. ACM, 2807-2816.

[38] Meizi Zhou, Zhuoye Ding, Jiliang Tang, and Dawei Yin. 2018. Micro behaviors: A new perspective in e-commerce recommender systems. In WSDM. ACM, 727-735. 


\section{APPENDIX}

Table 4: Parameter Settings

\begin{tabular}{l|c}
\hline Parameter & Value \\
\hline Input Series length: & 128 \\
\# of Time Steps for Conv_LSTM: & 5 \\
Hidden State Dimensionality: & 32 \\
Embedding Dimension: & 32 \\
Batch Size: & 64 \\
Learning Rate: & $1 \mathrm{e}-3$
\end{tabular}

\subsection{Implementation Details}

For training/test data split, we use the data from $01 / 01 / 2015$ to $01 / 01 / 2018$ for training, data from $01 / 02 / 2018$ to $01 / 15 / 2018$ as validation, and the data from $01 / 16 / 2018$ to $06 / 30 / 2018$ is used for testing. We implemented all the deep learning baselines and the proposed GMP framework with Tensorflow ${ }^{1}$. For training models, we divided the datasets into training, validation and testing set in chronological order. The validation set is used to select the best parameter values. For the sake of fair comparison, all prediction experiments are conducted across the consecutive time steps (i.e., day) in the test data (refer to data descriptions for details) and the average performance is reported.

\subsection{Parameter Settings}

In our experiments, we set the dimension of hidden representation and sequence length in convolutional recurrent encoder as 32 and 5 , respectively. We have the channel size of $\left[2^{2}, 2^{4}, 2^{5}, 2^{6}\right]$ which corresponds to the process of high-level feature map generation (with a larger latent representation space). The number of channels in the top-down modulation network is set as 32. During the model learning process, we used the Adam optimizer for gradient-based model optimization, where the batch size and learning rate were set as 64 and 0.001 , respectively.

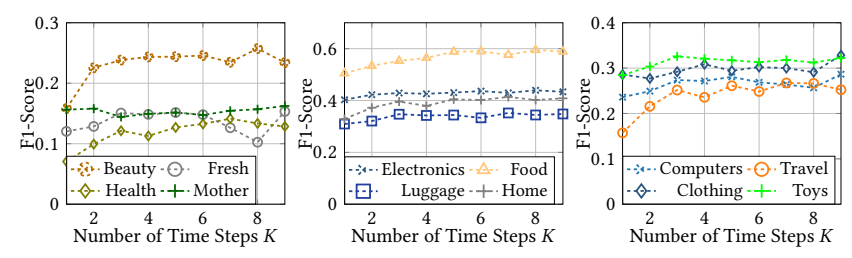

Figure 10: Hyperparameter Studies w.r.t the number of time steps $K$. Categories with similar performance range are presented in the same figure.

\subsection{Hyperparameters Studies (Q6)}

Figure 10 and Figure 11 show the evaluation results with different hyperparameter selections. From the evaluation results, we could observe that our method GMP is not very sensitive to these parameters. To be more specific, as $K$ increases, the prediction performance remains stable when $K=5$. One potential reason is that

\footnotetext{
${ }^{1}$ The code is available at https://github.com/graphmp
}
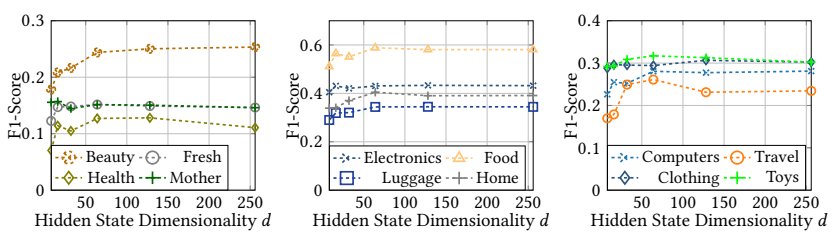

Figure 11: Hyperparameter Studies w.r.t hidden state dimensionality $d$. Categories with similar performance range are presented in the same figure.

when considering larger sequence length, more parameters need to be learned in the recurrent network architecture. In our experiments, we set $K=5$. Furthermore, we set the dimension size as 64 , due to the consideration of the trade-off between the effectiveness and computational cost. 